\title{
A INSUFICIÊNCIA RENAL AGUDA E TACROLIMUS APÓS TRANSPLANTE HEPÁTICO
}

\author{
Acute renal failure and tacrolimus after Liver Transplantation
}

\author{
Lucas Souto Nacif, André Ibrahim David, Marcio Augusto Diniz, Alessandra Crescenzi, Wellington Andraus, \\ Rafael Soares Pinheiro, Ruy Jorge Cruz, Luiz Carneiro D’Albuquerque
}

\section{RESUMO}

Introdução: O transplante de fígado melhorou consideravelmente a sobrevivência em pacientes com doença hepática em estágio final. Mas, continua sendo um desafio alcançar a terapia imunossupressora ideal, evitando complicações precoces e a insuficiência renal. Objetivo: Avaliar o nível sérico de tacrolimus com insuficiência renal aguda precoce após transplante de fígado, nos primeiros 30 dias de internação ou até a alta. Método: Foram estudados dados clínicos e laboratoriais dos pacientes submetidos a transplante de fígado, a partir de outubro de 2011 até fevereiro de 2013. Foram excluídos casos com doador vivo, hepatite aguda grave, fígado bipartido, uso de outros imunossupressores além da rotina (tacrolimus e prednisona), muito grave e que tenha morrido antes de 30 dias. A análise estatística foi realizada utilizando medidas descritivas (média \pm desvio padrão) das variáveis: níveis séricos de tacrolimus (ng/ml), taxa de filtração glomerular (TFG) $(\mathrm{ml} / \mathrm{min})$ e creatinina $(\mathrm{mg} / \mathrm{dl})$. Comparação das variáveis pela análise de variância (ANOVA) com medidas repetidas pelo programa estatístico não paramétrico em $R$, versão 2.15.1. Significância estatística com $p<0,05$. Resultado: Alta prevalência de pacientes do sexo masculino $(68,18 \%)$. A idade média dos pacientes foi de $52,43( \pm 12,33)$ e mediana de 55,5 (variação, 19-71 anos). O tempo médio de internação foi de $16,1 \pm 9,32$ dias. A causa principal para o transplante foi cirrose pelo vírus da hepatite $C(47,7 \%)$. A média do escore MELD foi de 26,18 $\pm 4,28$. A análise dos gráficos ao longo do tempo mostrou uma correlação significativa entre o valor sérico do tacrolimus, com a deterioração da taxa de filtração glomerular bem como da creatinina sérica. $\mathrm{Na}$ análise comparativa, observou-se diferença estatística em relação aos níveis séricos de tacrolimus e infecção $(p=0,0391)$ e em relação ao tempo $(p=0,0001)$. Notamos que na população estudada, $11,37 \%$ dos casos apresentaram rejeição celular aguda e $36,37 \%$ dos casos, infecção. Conclusão: O nível sérico de tacrolimus, quando superior a $10 \mathrm{ng} / \mathrm{ml}$, apresenta deterioração da taxa de função renal.

Descritores: Transplante de Fígado; Insuficiência Renal; Tacrolimo.

\section{Instituição:}

Disciplina de Transplante de Fígado e Órgãos do Aparelho Digestivo, Departamento de Gastroenterologia da FMUSP - São Paulo/SP, Brasil

\section{Correspondência:}

Dr. Lucas Souto Nacif

Rua Dr. Enéas de Carvalho Aguiar, 255 - s/9113/4 - CEP 05403-900 São Paulo/SP, Brasil

E-mail: lucasnacif@usp.br

Tel: (11) 2661-3323 Fax: (11) 2661-9008

\section{INTRODUÇÃO}

O transplante de fígado (TF) melhorou consideravelmente a sobrevivência em pacientes com doença hepática em estágio terminal. Esse sucesso foi em grande parte devido às melhorias na técnica cirúrgica, anestesia e eficácia da terapia imunossupressora com inibidores da calcineurina (ICN), como a ciclosporina e o tacrolimus. ${ }^{1,2}$

Com o uso dos inibidores de calcineurina (ICN), observouse melhora dramática na sobrevida do paciente após o transplante hepático; no entanto, apresentaram-se relatos de nefrotoxicidade em seu uso no pós TF. ${ }^{2,3}$

A insuficiência renal é um verdadeiro desafio para os médicos no pós TF. ${ }^{4}$ Com isso, a escolha da terapia imunossupressora pode influenciar, tanto a função renal como as complicações precoces após TF. 
Lucas Souto Nacif; André Ibrahim David; Marcio Augusto Diniz; Alessandra Crescenzi; Wellington Andraus; Rafael Soares Pinheiro; Ruy Jorge Cruz; Luiz Carneiro D’Albuquerque

Tendo em vista os dados acima, o objetivo deste estudo foi avaliar o nível sérico de tacrolimus na insuficiência renal aguda precoce após TF, nos primeiros 30 dias de internação ou até a alta hospitalar.

\section{MÉTODOS}

\section{Desenho do Estudo}

Foram estudados dados clínicos e laboratoriais de 44 pacientes submetidos a TF, entre outubro de 2011 e fevereiro de 2013; foram excluídos aqueles com doador vivo, hepatite aguda grave, split fígado, em uso de outros imunossupressores além dos de rotina (tacrolimus e prednisona), muito grave e que morreram antes de 30 dias. A taxa de filtração glomerular (TFG) foi estimada de acordo com a equação de Cockcroft-Gault. ${ }^{5}$

\section{Técnica cirúrgica e imunossupressão}

$\mathrm{Na}$ maioria dos casos, os procedimentos foram realizados com a técnica piggyback; a solução de preservação utilizada foi Belzer (UW). O protocolo de imunossupressão foi utilizado para todos os pacientes, com bolus de esteróides durante a fase de anepática e introdução via oral com o objetivo de parar por completo depois de três a seis meses após o TF. O inibidor de calcineurina (tacrolimus) foi iniciado entre o primeiro e terceiro dia, na dose 0,10 a $0,15 \mathrm{mg} / \mathrm{kd} / \mathrm{dia}$, administrada via oral e mantida em todos os pacientes; o micofenolato de mofetil (MMF) foi empregado em pacientes com indicação de transplante duplo fígado/rim ou disfunção renal prévia. Os episódios de rejeição celular aguda foram confirmados por biópsia, respeitando-se os critérios de Banff e as infecções bacterianas, fungos ou virais, confirmadas por culturas.

\section{Análise estatística}

A análise estatística foi realizada utilizando-se medidas descritivas (média \pm desvio padrão) das variáveis: níveis séricos de tacrolimus (ng/ml), taxa de filtração glomerular (TFG) $(\mathrm{ml} / \mathrm{min})$ e creatinina $(\mathrm{mg} / \mathrm{dl})$. Comparação das variáveis por análise de variância (ANOVA) com medidas repetidas pelo programa estatístico não paramétrico em $\mathrm{R}$, versão 2.15.1. Os valores foram considerados estatisticamente significantes, com $\mathrm{p}<0,05$.

\section{RESULTADOS}

\section{Perfil Clínico e Demográfico}

Alta prevalência de pacientes do sexo masculino $(68,18 \%)$, idade média dos pacientes de $52,43( \pm 12,33)$ e mediana de 55,5 (variação, 19-71 anos). Tempo médio de internação de 16,1 \pm 9,32 dias. A causa principal para o transplante foi cirrose pelo vírus da hepatite C (47,7\%). A média do escore MELD (funcional) nos pacientes foi de $26,18 \pm 4,28$. Nos pacientes estudados, observamos $11,37 \%$ casos de rejeição celular aguda e $36,37 \%$ casos de infecção. (Tabela 1)
Tabela 1: Dados demográficos dos Transplantes de Fígado incluídos neste trabalho

\begin{tabular}{ll}
\hline Parâmetros & Transplante de Fígado (n=44) \\
\hline Sexo (n/\%) & $\mathrm{M}=30(68.18 \%) / \mathrm{F}=14(31.82 \%)$ \\
Idade média (anos) & $52.43( \pm 12.33)$ \\
Mediana idade (anos) & 55.5 (range, 19-71) \\
Média hospitalização (dias) & $16.1 \pm 9.32$ \\
MELD escore & $26.18 \pm 4.28$ \\
Etiologia & Cirrose VHC (47.7\%) \\
Rejeição Aguda & $\mathrm{n}=5(11.37 \%)$ \\
Infecção & $\mathrm{n}=16(36.37 \%)$ \\
\hline
\end{tabular}

Média e desvio padrão ou número e porcentagens; M: masculino, F: feminino; VHC: cirrose pela hepatite C; MELD: Modelo de Estágio Final de Doença Hepática.

\section{Dados Comparativos - Falência Renal}

A análise dos gráficos ao longo do tempo mostrou correlação significativa entre o valor do nível sérico do tacrolimus, com a deterioração da taxa de filtração glomerular (Figura 1), bem como da creatinina sérica (Figura 2).

Figura 1: Correlação gráfica pelo tempo do nível sérico de tacrolimus com a taxa de filtração glomerular

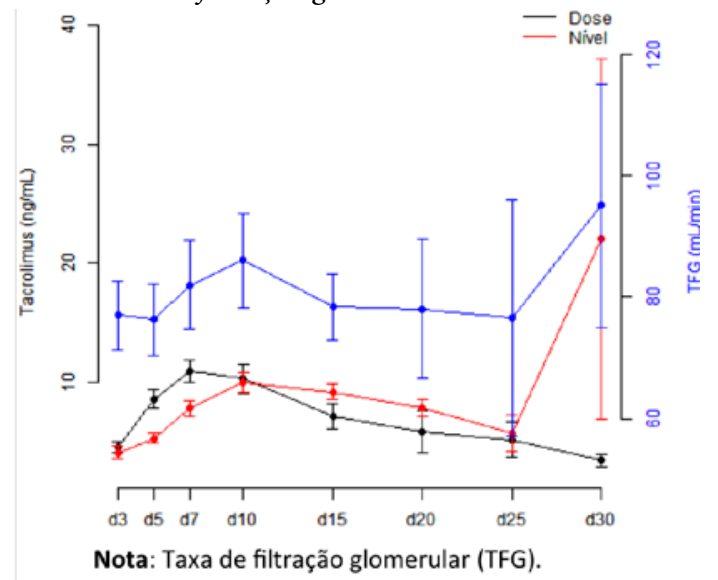

Figura 2: Correlação gráfica pelo tempo do nível sérico de tacrolimus com o nível sérico da creatinina

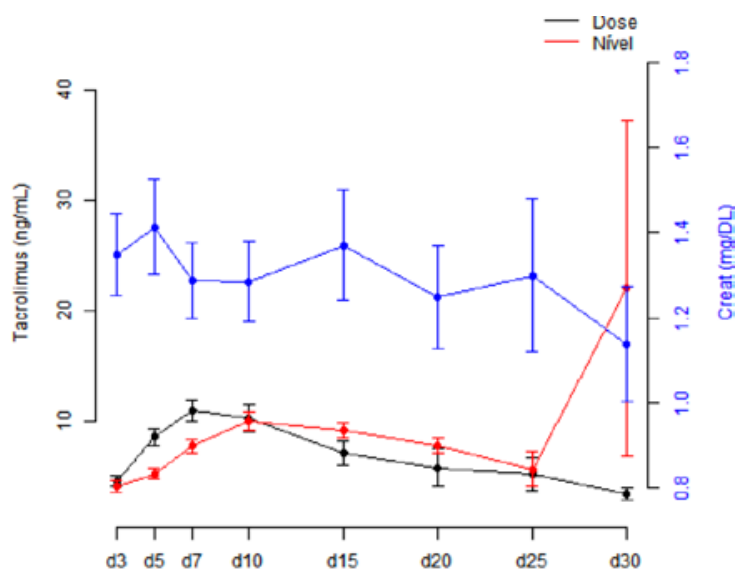

JBT J Bras Transpl. 2013;16(1):1715-1741 
$\mathrm{Na}$ análise comparativa, observou-se diferença estatística em relação aos níveis séricos de tacrolimus e infecção $(\mathrm{p}=0.0391) \mathrm{e}$ em relação ao tempo ( $\mathrm{p}=0,0001)$ (Tabela 2). Existem diferenças entre os valores médios de TFG para os grupos com e sem rejeição aguda $(\mathrm{p}=0,0084)$ e em relação ao nível de tacrolimus $(\mathrm{p}=0,0001)$ (Tabela 2).

Tabela 2: Comparação das variáveis estudadas nos grupos infecção e rejeição aguda.

\begin{tabular}{llcc}
\hline Variável & & Infecção (p valor) & Rejeição (p valor) \\
\hline Nível & Grupo & 0.4102 & $\mathbf{0 . 0 0 0 1}$ \\
Nível & Tempo & $\mathbf{0 . 0 0 0 1}$ & 0.1520 \\
Nível & Grupo: Tempo & $\mathbf{0 . 0 3 9 1}$ & 0.6876 \\
TFG & Grupo & 0.1450 & $\mathbf{0 . 0 0 8 4}$ \\
TFG & Tempo & 0.6142 & 0.4505 \\
TFG & Grupo: Tempo & 0.5685 & 0.5685 \\
Creat & Grupo & 0.2448 & 0.5695 \\
Creat & Tempo & 0.5726 & 0.4050 \\
Creat & Grupo: Tempo & 0.3264 & 0.5273 \\
\hline
\end{tabular}

Taxa de filtração glomerular (TFG) $(\mathrm{ng} / \mathrm{ml})$; Creatinina (creat) $(\mathrm{mg} / \mathrm{dl})$; nível sérico de tacrolimus $(\mathrm{ng} / \mathrm{ml})$.

\section{DISCUSSÃO}

$\mathrm{Na}$ análise dos gráficos de variância, ao longo do tempo, observamos correlação significativa entre o nível sérico de tacrolimus e a deterioração da taxa de filtração glomerular.

Com o objetivo de determinar a menor concentração sérica de tacrolimus recomendada, evitando piora da função renal, foi realizada uma revisão sistemática e meta-análises. ${ }^{3}$
As concentrações mínimas de tacrolimus $(<10 \mathrm{ng} / \mathrm{mL})$ no primeiro mês após o TF foram associadas com menor comprometimento renal no primeiro ano. ${ }^{3}$ Neste estudo, observou-se a mesma correlação do nível de tacrolimus com a TFG.

Podemos notar que a curva da TFG segue a curva do nível Tacrolimus. No momento em que os níveis séricos de tacrolimus estavam $>10 \mathrm{ng} / \mathrm{ml}$, por volta do décimo dia pós TF, observamos piora significativa da TFG, seguida por melhora progressiva com a diminuição do nível sérico do tacrolimus.

A alteração renal é fator conhecido no pós-transplante de fígado imediato, precoce e tardio; sabemos, com base em publicações, que essa alteração imediata está relacionada ao trauma cirúrgico, perda sanguínea e à modalidade do transplante realizado; a piora renal persistente pode ser decorrente de paciente previamente com algum grau de nefropatia ou em hemodiálise, pelo qual sua alteração renal acaba por se agravar com o pós-transplante e o uso da imunossupressão. Finalmente, no pós-operatório tardio, observamos a piora da função renal, principalmente devido ao agravamento renal pelo inibidor de calcineurina. ${ }^{3,4}$

Kong et al mostram-nos que, em pacientes com minimização ICN (dose mais baixa), observa-se diminuição significativa do nível sérico da creatinina. Os episódios de rejeição aguda e a sobrevida dos pacientes foram similares, mas podendo ser acompanhados por infecções. ${ }^{3}$ Concentrações mais baixas do tacrolimus (6-10 $\mathrm{ng} / \mathrm{mL}$, durante o primeiro mês), seria o mais adequado, após o TF.,

\section{CONCLUSÃO}

Piora da função renal quando o nível sérico de tacrolimus foi superior a $10 \mathrm{ng} / \mathrm{ml}$.

\section{ABSTRACT}

Introduction: Liver transplantation (LT) has considerably improved survival in patients with end-stage liver disease. But it remains a challenge to achieve optimal immunosuppressive therapy avoiding early complications and renal failure. Purpose: To evaluate the serum level of tacrolimus with early acute renal failure after liver transplantation in the first 30 days of admission or until discharge. Method: We studied clinical and laboratory data from patients who underwent liver transplantation from October 2011 to February 2013. It was excluded those from living donor, acute hepatitis, split liver, using other immunosuppressants besides routine (tacrolimus and prednisone), those severe ill and who died before 30 days. Statistical analysis was performed using descriptive measurements (mean \pm standard error) of variables: tacrolimus serum levels (ng/ml), glomerular filtration rate (GFR) $(\mathrm{ml} / \mathrm{min}$ ) and creatinine $(\mathrm{mg} / \mathrm{dl})$. Comparison of variables by analysis of variance (ANOVA) with repeated measures nonparametric statistical program $R$, version 2.15.1. Statistically significant at $p<0.05$. Result: We observed a high prevalence of male patients $(68.18 \%)$. The average age of patients was $52.43( \pm 12.33)$ and median of 55.5 (range, 19-71 years). The mean hospital stay was $16.1 \pm 9.32$ days. The main cause for liver transplantation was hepatitis C (47.7\%). The mean MELD score for patients was $26.18 \pm 4.28$. Graphic analysis over time showed a significant correlation between the tacrolimus serum value, with deterioration of the glomerular filtration and serum creatinine rates. The comparative analysis showed a statistical difference in relation to serum levels of tacrolimus and infection $(\mathrm{p}=0.076)$ and with respect to time $(\mathrm{p}=0.0001)$. We note that in the studied population $11.37 \%$ of the acute cellular rejection cases and $36.37 \%$ of the cases of infection. Conclusion: The serum level of tacrolimus when exceeding $10 \mathrm{ng} / \mathrm{ml}$ causes a deterioration of renal function rate.

Keywords: Liver transplantation; Renal Insufficiency; Tacrolimus 


\section{Agradecimentos}

Ao Laboratório de Epidemiologia e Estatística do Departamento de Gastroenterologia do Hospital das Clínicas da Faculdade de Medicina da Universidade de São Paulo.

\section{REFERÊNCIAS}

1. Sanchez EQ, Melton LB, Chinnakotla S, Randall HB, McKenna GJ, Ruiz R, et al. Predicting renal failure after liver transplantation from measured glomerular filtration rate: review of up to 15 years of follow-up. Transplantation. 2010;89(2):232-5.

2. Gonwa TA, Mai ML, Melton LB, Hays SR, Goldstein RM, Levy $\mathrm{MF}$, et al. End-stage renal disease (ESRD) after orthotopic liver transplantation (OLTX) using calcineurin-based immunotherapy: risk of development and treatment. Transplantation. 2001;72(12):1934-9.

3. Kong Y, Wang D, Shang Y, Liang W, Ling X, Guo Z, et al. Calcineurin-inhibitor minimization in liver transplant patients with calcineurin-inhibitor-related renal dysfunction: a metaanalysis. PLoS One. 2011;6(9):e24387.
4. MR, Wall WJ, Ojo AO, Ginès P, Textor S, Shihab FS, et al. Report of the first international liver transplantation society expert panel consensus conference on renal insufficiency in liver transplantation. Liver Transpl. 2009;15(11):S1-34.

5. Teruel, JL, et al. The Cockroft-Gault equation is better than the MDRD equation for estimating glomerular filtration rate in patients with advanced chronic renal failure. Nefrologia. 2007;27(3):313-9. 